\title{
A Study of Phage-Resistant Mutants of Rhizobium trifolii
}

\author{
BY J. KLECZKOWSKA \\ Soil Microbiology Department, Rothamsted Experimental Station, \\ Harpenden, Hertfordshire
}

SUMMARY: Phage-resistant mutants were obtained from four strains of Rhizobium trifolii. Mutation to phage resistance tended to coincide with mutations in other features, such as morphology of colonies or effectiveness in nitrogen fixation. The accompanying mutations occurred independently, and their frequency varied widely from strain to strain.

Some mutants seemed stable in their newly acquired features; others continued to mutate at high rates.

Some of the mutants as regards nitrogen fixation were stable or could eventually be stabilized, but other mutants derived from one of the parent strains remained unstable even after several successive replatings or passages through nodules.

Strains of Rhizobium belonging to the same inoculation group, i.e. capable of forming root nodules on the same species of leguminous plant, can differ from one another in various respects, such as colonial morphology, serological specificity, effectiveness of nitrogen fixation in root nodules, and ability to ferment different carbohydrates. Individual strains are usually stable, maintaining these features unaltered during many years of cultivation on artificial media. Under ordinary conditions of laboratory cultivation variants with new properties appear only rarely and irregularly. Much work has been done to find conditions in which variants can be obtained more frequently and regularly. Thus Nobbe \& Hiltner (1893) and Frank (1899) found that prolonged cultivation on gelatin could lead to a decreased effectiveness in nitrogen fixation; Simon (1908), Hutchinson (1924) and Snieszko (1929) found that the lost effectiveness could be restored by cultivation in soil. On the other hand, by prolonged storage in soil Nutman (1946) obtained ineffective variants from effective strains, but not vice versa.

Allen \& Baldwin (1931) obtained more effective variants from less effective strains and vice versa by passages through host plants, but their results did not agree with those of Stapp (1929), and could not be reproduced by Virtanen (1945) or Nutman (1946).

The disagreements between different workers show that the treatments which they described do not regularly give the same results. The divergencies can be explained by assuming that different strains produce mutants which differ in their response to the selective action of a given treatment.

The morphology of colonies of Rhizobium strains is another feature whose changes were studied. Almon \& Baldwin (1933) obtained variants whose colonies differed from those of parent strains in various respects, some showing yellow or pink pigmentation. Various methods, including treatment with bacteriophage, were used to obtain such variants.

Treatment with a bacteriophage is a rapid means of isolating mutants that are resistant to it. There is evidence that bacterial strains produce phage- 
resistant mutants independently of the action of the phage, which merely reveals their presence by destroying susceptible organisms (Burnet, 1929; Luria \& Delbrück, 1948; Kleczkowska, 1945b; Newcombe, 1949). Fresh mutants will probably be unstable in more respects than one, and so mutations in other features can be expected, at least occasionally, to accompany mutations in susceptibility to a phage. They may include mutations in morphology of colonies (Almon \& Baldwin, 1938), and mutations in ability and efficiency to form nodules (Krasil'nikov, 1941).

The purpose of the present work was to study the mutations in colonial morphology and in effectiveness of nitrogen fixation that accompany mutations in phage susceptibility, and to see whether they tend to occur together.

\section{MATERIALS AND METHODS}

The four strains of $R h$. trifolii used were:

Strain $A$, effective in nitrogen fixation.

Strain $B$, an ineffective variant obtained by Nutman from strain A by storage in soil and designated as $f 12$ (Nutman, 1946). Strains A and B were identical serologically.

Strains $C$ and $D$ (= strains $C$ and HKC of Nutman, 1946) were both ineffective nitrogen fixers. They were serologically unrelated to each other and to strains $A$ and $B$.

All the four strains formed greyish white opaque colonies of smooth type, those of strain $\mathbf{C}$ being more slimy than the others. The four strains maintained these features unaltered during several years' cultivation on laboratory media and after several passages through host plants.

Two different bacteriophages, both isolated from the soil and maintained in liquid culture as previously described (Kleczkowska, 1945a), were used. Strains $A$ and $B$ were treated with phage $S_{2} P_{11}$ and strains $C$ and $D$ with phage $C l_{15}$. Phage $S_{2} P_{11}$, which formed large-sized plaques, could lyse strains $A$ and $B$ but not $\mathrm{C}$ and $\mathrm{D}$, whereas phage $C l_{15}$, which formed small-sized plaques, could lyse all four strains. Phage $\mathrm{Cl}_{15}$ was not used with all four strains because the work was started with strains $A$ and $B$ and phage $S_{2} P_{11}$ when phage $C l_{15}$ was not available.

The agar medium used for cultivating the bacterial strains, the nitrogendeficient 'seedling agar' used for growing clover plants in sterile conditions in test tubes, and the technique of growing the plants and of re-isolating bacteria from root nodules, were as described by Nutman (1946).

Late flowering Montgomery red clover was used as host plant. The plants were infected by bacterial strains under test by inoculating the surface of the 'seedling agar' at the time when the first true leaf was opened. Each tested strain was inoculated into a number of tubes each containing two plants. The plants were allowed to grow for about 3 months until eight true leaves were formed on control plants infected with a known strain fully effective in nitrogen fixation. A strain was classified as 'effective' if test plants inoculated with it developed as abundantly as those inoculated with the control effective strain. A strain was classified as 'ineffective' if the test plants showed as poor develop- 
ment as the uninoculated control plants; otherwise a strain was classified as 'intermediate'.

The bacterial strains were treated with bacteriophage by suspending a loopful of cells, taken from a fresh culture on agar slope, in $1 \mathrm{ml}$. of liquid phage culture, shaking the suspension for a few minutes to homogenize it, and spreading four drops of the suspension on the surface of an agar plate. The plates were incubated at $25^{\circ}$ for 12 days. At first there was no visible bacterial growth, because all phage-susceptible organisms were lysed. On the eighth to tenth day single colonies of resistant cells appeared, and by the twenty-first day their growth was sufficient for isolation. After isolation, the growth of the strains was apparently as vigorous as that of the parent strains, i.e. their colonies would be well developed in about 5 days.

\section{RESULTS}

\section{The appearance of new features}

Fifteen to twenty plates were used with each of the four bacterial strains for the isolation of bacteria that had survived the phage treatment. All isolations were made from single colonies. The numbers of colonies from which isolations were made were $50,57,55$ and 55 from strains $A, B, C$ and $D$ respectively. Eight strains isolated from phage-treated strain $B$ appeared to be unable to induce nodule formation. Such 'non-infective' mutants have been isolated previously by means of phage treatment from normal nodule-producing strains, and described by Krasil'nikov (1941). In the present work these non-infective mutants were not studied in any detail, and only those that retained the ability to form nodules are dealt with below. The fact that non-infective mutants were obtained only from one of the four tested strains suggests that the tendency to form such mutants varies from one strain to another.

Some of the phage-resistant colonies did not differ in appearance from those of the parent strains, others differed in various respects. Some were typically rough, others smooth or intermediate; some were opaque and others transparent; some were pure white whereas others showed yellow or pink pigmentation. The colonies were composed of Gram-negative rods that did not differ in appearance from those of the parent strains, except for the presence of Gram-positive granules in the rods of most colonies which showed yellow or pink pigmentation.

Table 1 gives the numbers of morphologically new and parent-type colonies obtained from the four tested strains. The figures in brackets show the numbers

Table 1. The numbers of morphologically changed and unchanged colonies

(The numbers in brackets are computed by distributing the right-hand marginal totals in proportion to the bottom-line totals. $\chi^{2}=27.5 ;$ D.F. $=3 ; P<0.001$.)

$\begin{array}{cccc}\text { Parent strain } & \text { Unchanged } & \text { Changed } & \text { Total } \\ \text { A } & 4(13) & 46(87) & 50 \\ \text { B } & 15(13) & \mathbf{3 4}(\mathbf{3 6}) & 49 \\ \text { C } & 9(15) & 46(40) & 55 \\ \text { D } & 28(15) & 27(40) & 55 \\ & 56 & 153 & 209\end{array}$


of new and parent type colonies expected if all four strains produced the same percentage of new types. These were calculated by dividing each total shown in the right column in proportion to those in the bottom line. The actual numbers differ from those expected with a $\chi^{2}$ of 27.5 with 3 degrees of freedom $(P<0.001)$, showing that the four strains differ very significantly in their tendency to produce colony mutants.

All the strains isolated from the forty-nine colonies from strain B were tested serologically with an antiserum to the parent strain. Four of them failed to agglutinate.

The results of testing the derivative strains for effectiveness in nitrogen fixation (Table 2) are based on four replicate tubes and show that the four parent strains gave widely different proportions of effective and ineffective mutants. A large proportion of derivatives obtained from the effective

Table 2. Numbers of derivative strains classified according to their effectiveness in nitrogen fixation

(Strains classified as uniform produced the same response (effective or ineffective) on all test plants used. Those classified as non-uniform gave different results in the four replicate tubes. The numbers of effective, intermediate and ineffective responses in the individual tubes in this group were respectively 18, 26, 44 for strain $A ; 3,5,20$ for strain $B ;$ and $1,4,15$ for strain C.)

$\begin{array}{lcccc}\text { Parent strain } & \overbrace{\text { Effective }} \text { Uniform } & \text { Ineffective } & \begin{array}{c}\text { Non- } \\ \text { uniform }\end{array} & \begin{array}{c}\text { Total } \\ \text { numbers }\end{array} \\ \text { A (effective) } & \mathbf{1 7} & 11 & 22 & 50 \\ \text { B (ineffective) } & 1 & 41 & 7 & 49 \\ \text { C (ineffective) } & 0 & 50 & 5 & 55 \\ \text { D (ineffective) } & 0 & 55 & 0 & 55\end{array}$

strain A differed from the parent strain; only small proportions of derivatives obtained from the ineffective strains $\mathrm{B}$ and $\mathrm{C}$ differed from them, and all the derivatives obtained from the ineffective strain $D$ remained unchanged. It is obvious, therefore, that the tendency to produce mutation in nitrogen fixing effectiveness varies very considerably from one strain to another. Some of the derivative strains behaved uniformly, i.e. gave the same results on all test plants whereas some others did not, i.e. gave different results on different test plants. Experiments designed to examine this non-uniformity are described below.

When the derived strains were tested with the bacteriophage used for their isolation it was found that some were lysed (Table 3). About the same propor-

Table 3. The numbers of phage-resistant and susceptible derivatives

(The numbers in brackets computed as in Table $1 . \chi^{2}=2.53 ; D . F .=3 ; P=0.5$.)

\begin{tabular}{clcc} 
& \multicolumn{2}{c}{ Derivatives } & \\
\cline { 2 - 3 } Parent strain & Resistant & Susceptible & Totals \\
A & $41(38)$ & $9(12)$ & 50 \\
B & $36(37)$ & $13(12)$ & 49 \\
C & $38(41 \cdot 5)$ & $17(13 \cdot 5)$ & 55 \\
D & $43(41.5)$ & $12(13.5)$ & 55 \\
& 158 & 51 & 209
\end{tabular}


tions of derivates obtained from the four parent strains were phage-susceptible. The small value of $\chi^{2}$ shows that deviations of actual numbers from those computed on the assumption that all the strains producing resistant and susceptible derivatives in the same proportions are not significant.

The presence of phage-susceptible strains among the derivatives isolated by means of phage treatment could be explained as a result of local development of protective substances similar to those that were occasionally demonstrated

Table 4. The total numbers of derivatives with morphologically changed and unchanged colonies among phage-resistant and susceptible derivatives obtained from all four parent strains

(The numbers in brackets computed as in Table $1 . \chi^{2}=2 \cdot 7 ;$ D.F. $=1 ; P=0 \cdot 1$.)

\begin{tabular}{lcrc} 
& \multicolumn{2}{c}{ Morphology of colonies } & \\
\cline { 2 - 3 } Resistant & Unchanged & Changed & Totals \\
Susceptible & $47(42 \cdot 5)$ & $111(115 \cdot 5)$ & 158 \\
& $9(13 \cdot 5)$ & $42(37 \cdot 5)$ & 51 \\
& 56 & 153 & 209
\end{tabular}

Table 5. The total numbers of derivatives with changed and unchanged effectiveness in nitrogen fixation among phage-resistant and susceptible derivatives obtained from strains $A, B$ and $C$

(The numbers in brackets computed as in Table $1 . \chi^{2}=1.06 ;$ D.F. $=1 ; P=0.3$. )

\begin{tabular}{lcrr} 
& \multicolumn{2}{c}{ Nitrogen fixation } & \\
\cline { 2 - 3 } Resistant & Unchanged & Changed & Totals \\
Susceptible & $79(81.5)$ & $36(33 \cdot 5)$ & 115 \\
& $30(27 \cdot 5)$ & $9(11 \cdot 5)$ & 39 \\
& 109 & 45 & 154
\end{tabular}

in liquid cultures containing phage-susceptible secondary growths (Kleczkowska, $1945 b$ ). The presence of such protective substances would enable bacteria identical with those of the parent strains to survive and to form colonies. An alternative explanation is that the colonies were composed of bacteria immune to the phage, but the immunity was lost in the course of further growth after the isolations. The second explanation seems to be more probable for two reasons. First, the phage-susceptible derivatives were not identical with the parent strains, for they included forms that differed from the parent strains in colonial morphology and effectiveness of nitrogen fixation. Moreover, Tables 4 and 5 show that approximately the same proportions of derivatives changed and unchanged in colonial morphology and effectiveness in nitrogen fixation, were phage-resistant and susceptible. Secondly, although some of the derivative strains were phage-susceptible, they differed from the parent forms in that their complete lysis took longer and the secondary growth appeared much sooner.

Table 6 showed that mutants in colonial morphology were equally distributed among strains that did and did not mutate in effectiveness of nitrogen fixation. The two mutations, therefore, occurred independently of each other. 
Table 6. The total numbers of derivatives with changed and unchanged effectiveness in nitrogen fixation among those with changed and unchanged morphology of colonies, obtained from strains $A, B$ and $C$

(The numbers in brackets computed as in Table 1. $\chi^{2}=0.86 ;$ D.F. $=1 ; P=0.35$.)

Morphology
of colonies
Unchanged
Changed

Nitrogen fixation

$\begin{array}{ccc}\text { Unchanged } & \text { Changed } & \text { Totals } \\ 21(19) & 6(8) & 27 \\ 88(90) & \mathbf{3 9}(\mathbf{3 7}) & 127 \\ 109 & 45 & 154\end{array}$

When the strains A, B, C and D were plated with bacteriophage to isolate phage-resistant mutants, they were also plated without bacteriophage. All the colonies (500-1000 in number from each strain) were alike in appearance, being greyish white, opaque and of smooth type. Isolations were made from a number of colonies equal to the number of colonies obtained at the same time by phage treatment, and the isolates were tested for effectiveness in nitrogen fixation and for susceptibility to the bacteriophages. All the isolates were identical with the strains from which they were obtained. It can be concluded, therefore, that the strains are normally stable in culture as regards the features under examination, and that it was the phage treatment that revealed the presence of the mutant forms.

\section{Stability of new features}

The new morphological features of colonies of derivative strains obtained by phage treatment remained unaltered during up to 3 years' propagation on the agar medium with subculture at 5-6 month intervals. On the other hand, after a single passage through the host plant, most of the strains acquired the colonial appearance of the parent strains from which they have been derived, although in some cases the new characters such as roughness and pink colour were retained after a passage through the nodule.

The newly acquired level of effectiveness in nitrogen fixation was usually retained unaltered during cultivation on media as well as during passages through host plants, by those strains which behaved uniformly when tested the first time, i.e. which were either effective or ineffective on all test plants used. Table 2 shows, however, that the proportion of these uniform derivative strains was rather small. Others behaved non-uniformly, i.e. a proportion of plants on which they were tested responded effectively, some intermediately and the remainder ineffectively. There were, for example, no derivatives from strain $\mathbf{C}$ that differed from it in effectiveness and which behaved uniformly.

Strains behaving non-uniformly can be conveniently divided into two groups according to whether uniformly behaving cultures could be isolated from them. All the non-uniform strains from strains A and B belonged to the first group. The cultures isolated from plants that responded ineffectively were all uniformly ineffective. Those isolated from plants that responded effectively (or intermediately) were either all uniformly effective (or intermediate), or some, usually isolated from nodules deliberately chosen for their large size, were 
uniformly effective (or intermediate) and those isolated from nodules chosen for their small size, uniformly ineffective. This resembles segregation of mixtures into separate components. Isolations from single nodules usually give pure cultures of single strains when plants are inoculated with mixtures of different strains (Nicol \& Thornton, 1941; Hughes \& Vincent, 1942).

The five non-uniform strains derived from strain C (Table 2) all belonged to the second group. The cultures obtained from single nodules of the plants on which they were tested, irrespective of the character of their response, were

Table 7. Total numbers of intermediate and ineffective responses obtained with cultures isolated from each plant inoculated with a different strain derived from strain $C$

(The numbers in brackets computed as in Table $1 . \chi^{2}=8.9 ;$ D.F. $=4 ; P=0.065$.)

$\begin{array}{ccccc}\text { Number of } & & & \\ \text { Plant } & \begin{array}{c}\text { nodules } \\ \text { plated }\end{array} & \text { Intermediate } & \text { Ineffective } & \text { Totals } \\ 1 & 6 & 14(9 \cdot 6) & 46(50 \cdot 4) & 60 \\ 2 & 6 & 15(9 \cdot 6) & 45(50 \cdot 4) & 60 \\ 3 & 12 & 13(19 \cdot 2) & 107(100 \cdot 8) & 120 \\ 4 & 12 & 18(19 \cdot 2) & 102(100 \cdot 8) & 120 \\ 5 & 9 & 12(14 \cdot 4) & 78(75 \cdot 6) & 90 \\ & 45 & 72 & 378 & 450\end{array}$

again non-uniform in their behaviour. Table 7 illustrates the results of experiments leading to this conclusion. One plant showing an effective or intermediate response was chosen from each set of plants infected with each of the five derived strains that behaved non-uniformly. Platings were made from a number (6-12) of nodules from each plant and from each plating ten single colonies were tested on the plant for effectiveness. Columns 3 and 4 give the total numbers of intermediate and ineffective responses (there were no effective responses) obtained with all the colonies derived from nodules of each of the five plants. The cultures from different plants tended to give different ratios of intermediate to ineffective results, but the value of $\chi^{2}$ shows that this difference may have arisen by chance.

The results obtained individually with the forty-five sets of ten tests, in each of which a culture isolated from each single nodule was tested, are given in Table 8. The total numbers of sets that contained different numbers of plants which gave intermediate responses are shown. Out of the total of forty-five sets distributed amongst the five plants, thirteen gave ineffective responses in all ten tests and the remainder gave different kinds of responses.

Table 7 shows that $16 \%$ of the total of 450 tests gave intermediate responses. If, therefore, all the tested cultures had been identical, the probabilities of a test giving intermediate or ineffective response would be $0 \cdot 16$ and 0.84, respectively. Thus the probabilities that ten replicate tests will contain $0,1,2,3$, etc., plants responding intermediately would equal the successive terms of the expanded binomial $(0 \cdot 84+0 \cdot 16)^{10}$. The probabilities are given in Table 8, and the expected numbers of sets of ten plants, out of the total of 
Table 8. Total numbers of sets of ten plants containing different numbers of plants that gave intermediate responses

$\begin{array}{cc}\text { Number of } & \left(\chi^{2}=\mathbf{8} \cdot 94 ; \mathrm{D}\right. \\ \begin{array}{c}\text { intermediate } \\ \text { responses }\end{array} & \\ 0 & \text { Probability } \\ 1 & 0 \cdot 175 \\ 2 & 0 \cdot 333 \\ 3 & 0 \cdot 286 \\ 4 & 0 \cdot 145 \\ 5 & 0 \cdot 048 \\ 6 & 0 \cdot 011 \\ 7 & 0 \cdot 0017 \\ 8 & 0 \cdot 00018 \\ 9 & 0 \cdot 000013 \\ 10 & 0 \cdot 00000055 \\ & 0 \cdot 000000011\end{array}$

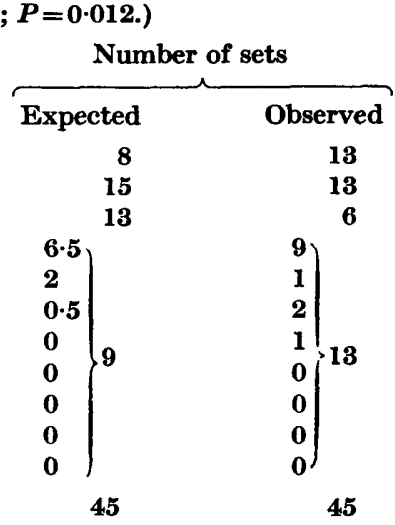

forty-five, that would contain different numbers of plants responding intermediately, are obtained from these probabilities. The value of $\chi^{2}$ for the deviations of actual from the expected numbers shows that the deviations are significant, and hence the chances of giving intermediate or ineffective response in a single test by different cultures were not identical.

Table 9. Total numbers of intermediate and ineffective responses obtained with cultures isolated from large and small nodules

(The numbers in brackets computed as in Table $1 . \chi^{2}=0.149 ;$ D.F. $=1 ; P=0.7$.)

$\begin{array}{lclc} & \text { Intermediate } & \text { Ineffective } & \text { Totals } \\ \text { Large } & \mathbf{3 7}(\mathbf{3 8} \cdot 5) & 203(\mathbf{2 0 1} \cdot 5) & 240 \\ \text { Small } & \mathbf{3 5}(\mathbf{3 3 \cdot 5 )} & 175(\mathbf{1 7 6} \cdot 5) & 210 \\ & 72 & 378 & 450\end{array}$

Table 9 shows that cultures isolated from nodules marked for their relatively large size did not tend to give results different from those given by cultures isolated from nodules marked for their relatively small size. On another occasion nodules developed during the winter with the same strain $\mathbf{C}$ attained a large size while still giving an ineffective response. Sections of these showed that their bacterial tissue was almost wholly disintegrated. (Unpublished observation of Dr P. S. Nutman.)

The non-uniform derivatives retained their diverse behaviour not only on plant passage but also after repeated platings. This is shown by experimental results given in Table 10. Two plants ( 1 and 2, Table 7), which had served as test plants in the experiment shown in Tables 7-10 to test two of the derived strains, were used as starting material. Ten nodules from each plant were plated and a loopful from each of ten colonies was tested for effectiveness, thus giving 100 tests from the nodules of each plant. These 100 tests are referred to as generation $\mathbf{I}$. 
Next, one colony isolate, derived from each of the nodules was plated again, and ten of the resulting colonies tested. This gave a second set of 100 tests referred to as generation II. This procedure was repeated once more, a third 'generation' of cultures being obtained (generation III), and these were similarly tested for effectiveness. Thus three successive platings were made from bacteria ultimately derived from each nodule. The experiment thus examines: (1) the

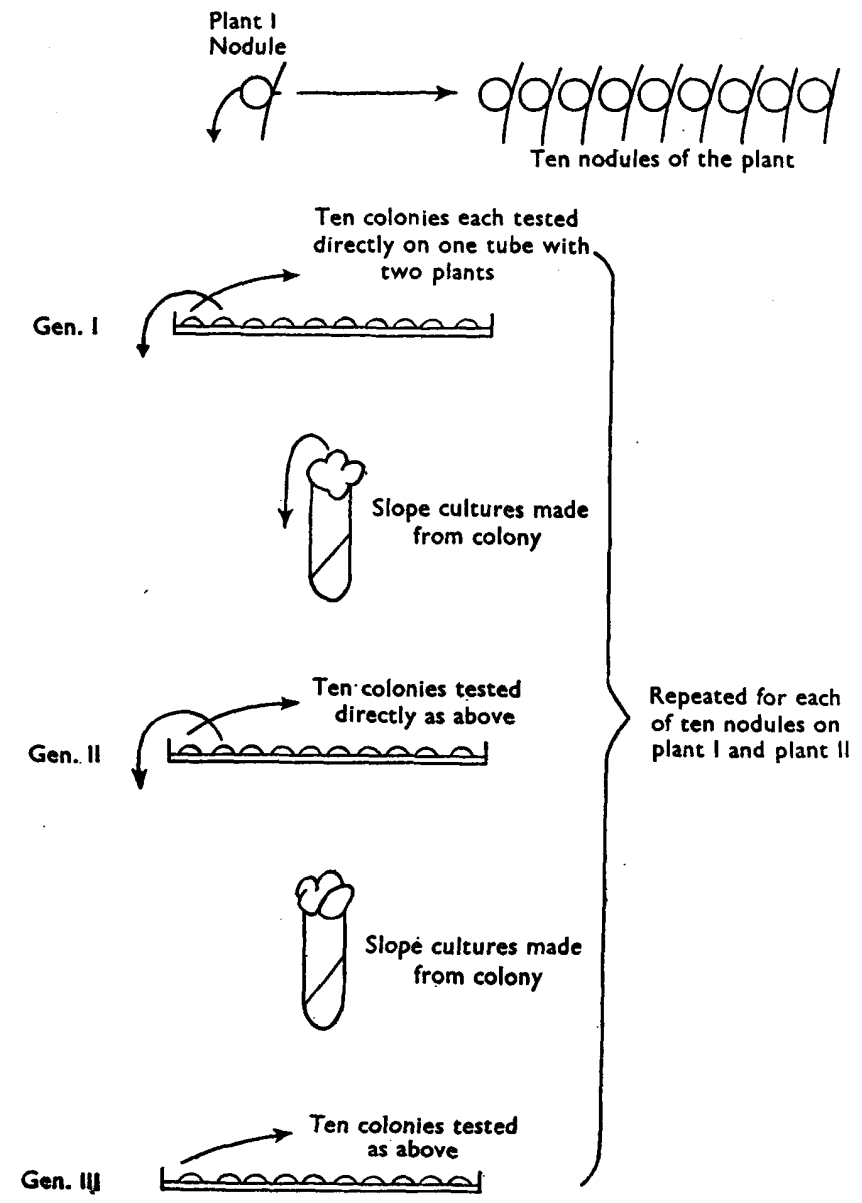

Fig. 1. Plan of the experiment given in Table 10

possibility of altering the proportion of parent and mutant types of bacteria by replating, and (2) the possible differences between the contents of individual nodules as regards the tendency to produce mutants.

The plan of this experiment is set out in the diagram (Fig. 1). Table 10, which gives the results, shows that there was considerable variation between derivatives from different nodules, but the variation between 'generations' was remarkably small. Statistical analysis (see Appendix) showed, however, that because of an interaction between nodules and 'generations' no direct conclu- 
Table 10. The results of testing isolates from single colonies obtained from non-uniform derivatives from strain $C$

(The numbers given are of 'intermediate' responses obtained in sets of ten tests with each isolate from a single nodule, the remainder of the set of ten responses being 'ineffective'. A proportion of responses were actually 'effective'. These are added to 'intermediate' responses and the totals are referred to as 'intermediate'.

\begin{tabular}{|c|c|c|c|c|c|c|c|c|c|c|c|}
\hline \multirow[b]{2}{*}{ 'Generation' } & \multicolumn{10}{|c|}{ Nodules } & \multirow[b]{2}{*}{ Totals } \\
\hline & i & 2 & $\mathbf{3}$ & 4 & 5 & 6 & 7 & 8 & $\mathbf{9}$ & 10 & \\
\hline \multicolumn{12}{|c|}{ Plant I } \\
\hline I & $\mathbf{3}$ & $\mathbf{0}$ & $\mathbf{3}$ & 5 & 1 & 4 & $\mathbf{3}$ & $\mathbf{1}$ & $\mathbf{3}$ & $\mathbf{1}$ & 24 \\
\hline II & 2 & 1 & 4 & 2 & 0 & 0 & 5 & $\mathbf{3}$ & 4 & 4 & 25 \\
\hline III & 4 & $\mathbf{3}$ & 5 & $\mathbf{0}$ & $\mathbf{3}$ & $\mathbf{1}$ & $\mathbf{0}$ & $\mathbf{3}$ & $\mathbf{2}$ & $\mathbf{3}$ & 24 \\
\hline Totals & 9 & 4 & 12 & 7 & 4 & $\mathbf{5}$ & 8 & 7 & $\boldsymbol{9}$ & 8 & 73 \\
\hline \multicolumn{12}{|c|}{ Plant II } \\
\hline $\mathbf{I}$ & 7 & 4 & 6 & $\mathbf{1}$ & 1 & $\mathbf{2}$ & $\mathbf{3}$ & 1 & $\mathbf{3}$ & 2 & 30 \\
\hline II & 4 & 1 & 5 & 5 & 2 & 2 & 4 & $\mathbf{5}$ & 4 & 1 & $\mathbf{3 3}$ \\
\hline III & 1 & 1 & $\mathbf{5}$ & 4 & $\mathbf{0}$ & $\mathbf{1}$ & $\boldsymbol{9}$ & $\mathbf{5}$ & 6 & 7 & $\mathbf{3 9}$ \\
\hline Totals & 12 & 6 & 16 & 10 & $\mathbf{3}$ & $\mathbf{5}$ & 16 & 11 & 13 & 10 & 102 \\
\hline
\end{tabular}

sion about the possibility of obtaining uniform behaviour can be drawn from the fact that generation totals varied only slightly. This could be the result of cancelling-out of opposite trends shown by derivatives from different nodules. It is apparent that cultures behaving uniformly could not be obtained by the replating.

In generation III, from plant II, some such possibility was suggested by two sets of ten colony isolates, which, on test, gave 0 and 9 effective responses. The two parent strains from generation III that gave these results were replated, and from each strain sixty colonies were tested. The numbers of effective, ineffective and intermediate responses with the first were 13, 12 and 35, and with the second 15, 17 and 28 . The value of the $\chi^{2}$ for deviations of these numbers from $14,14.5$ and 31.5 , which would be expected if the cultures were identical, is 1.78, which for the 2 degrees of freedom, corresponds to the probability of about $\mathbf{0 \cdot 4}$. Therefore the behaviour of the two strains was almost identical and none was uniform.

\section{DISCUSSION}

The results described above show that phage-resistant mutants tended to be more unstable than the parent strains with regard to various other properties. The newly acquired features remained stable with some mutant strains, but underwent rapid changes with others. An example of this is the rapid loss of immunity to bacteriophage in a proportion of mutant strains. There is also evidence that the strains that became sensitive again acquired immunity more readily than did the untreated strains, since secondary growth in liquid media developed much more rapidly than with the original strains.

The fact that after a single plant passage almost all mutant strains lost their newly acquired colony characters, although retaining them during several years' propagation on laboratory media, suggests a strong selection in the 
nodule tissue against colony mutant types. The mutant types differing from the parent strain in effectiveness in nitrogen fixation showed marked differences in stability. A number showed consistent behaviour amongst replicate isolates and retained their new character unaltered on laboratory media and after plant passage. Others did not show this uniform behaviour, and were of two types. Those obtained by phage treatment of strains $A$ and $B$ gave mixed responses at first, but after plant passage and re-isolation from single nodules they could be separated into derived strains that were consistent in giving effective or ineffective responses. This suggests that the original phage-resistant colonies contained mixed populations from which stable mutant types were isolated by plant passage and replating.

The second type of non-uniform derivatives, obtained by phage treatment of strain C, could not be purified either by re-isolation from nodules or by replating, but retained an unimpaired tendency to produce mutants giving effective or intermediate responses. After replating these derived strains, every colony tested was found to contain mutant forms in proportions that remained high after successive platings. Since an examination of the composition of a nodule involved plating the data do not enable us to tell how far mutant forms, which certainly appear in the colonies, also develop in the nodule tissue. The evidence does show, however, that these derived strains are highly unstable and that this instability is retained both on replating and on plant passage.

For reasons given previously (Kleczkowska, 1945b), it seems unlikely that phage can directly cause a failure of legume crops by destroying the nodule bacteria. It is possible, however, that phages may increase the proportion of ineffective strains in the soil under certain conditions and this possibility is enhanced by the observation that ineffective phage-resistant mutants are much more readily developed from effective parent strains than vice versa.

The author wishes to thank Dr H. G. Thornton, F.R.S., for his interest in this work and for aid in preparation of the manuscript and Dr A. Kleczkowski for statistical examination of the results.

\section{APPENDIX}

The proportions of 'intermediate' responses, shown in Table 10, were transformed into the angular degrees and subjected to analysis of variance, the results of which are given in Table 11. As ten tests were made with each 'generation' of each strain derived from a single nodule, the theoretical value of the variance is $820 \cdot 7 / 10=82 \cdot 07$. The 'residual' variance (obtained in the analysis of variance by subtracting from the total sum of squares of deviations from the mean those due to overall differences between derivatives from different nodules and between different 'generations'), is, in the case of both plants, significantly greater than the theoretical variance $(P<0 \cdot 001)$. This can be interpreted as an evidence of interaction between the origin from different nodules, on the one hand, and different 'generations', on the other. Thus, the tendencies to produce an 'intermediate' or 'ineffective' response probably 
Table 11. The analyses of variances of the results given in Table 10

(The proportions of 'intermediate' responses were transformed into angular degrees.)

\begin{tabular}{|c|c|c|c|c|c|}
\hline & \multirow[b]{2}{*}{ D.F. } & \multicolumn{2}{|c|}{ Plant I } & \multicolumn{2}{|c|}{ Plant II } \\
\hline & & $\begin{array}{c}\text { Sums } \\
\text { of } \\
\text { squares }\end{array}$ & $\begin{array}{c}\text { Mean } \\
\text { square } \\
\text { (variance) }\end{array}$ & $\begin{array}{c}\text { Sums } \\
\text { of } \\
\text { squares }\end{array}$ & $\begin{array}{c}\text { Mean } \\
\text { square } \\
\text { (variance) }\end{array}$ \\
\hline Between nodules & $\mathbf{9}$ & $1482 \cdot 95$ & $164 \cdot 77$ & $2919 \cdot 02$ & 324.34 \\
\hline Between 'generations' & $\mathbf{2}$ & $5 \cdot 21$ & $\mathbf{2 \cdot 6 0}$ & $\mathbf{8 8 . 2 0}$ & $44 \cdot 10$ \\
\hline Residual & 18 & $4586 \cdot 93$ & $254 \cdot 82$ & $4072 \cdot 07$ & $226 \cdot 23$ \\
\hline Total & 29 & $6075 \cdot 09$ & & $7079 \cdot 29$ & \\
\hline
\end{tabular}

changed from one 'generation' to another, differently with derivatives of different nodules, and, consequently, no direct conclusions can be drawn from 'generation' totals (of untransformed data or of the transformed proportions).

Variation between nodules is highly significant with derivatives from both plants $(\boldsymbol{P}=0.05$ for plant I and $\boldsymbol{P}<0.001$ for plant II).

\section{REFERENCES}

Allen, O. N. \& Baldwin, I. L. (1931). The effectiveness of Rhizobia as influenced by passage through the host plant. Bull. Wis. agric. Exp. Sta. no. 106.

Almon, L. \& Baldwin, I. L. (1933). The stability of cultures of Rhizobium. J. Bact. 26, 229.

BuRNET, F. M. (1929). Smooth-rough variation in bacteria in its relation to bacteriophage. J. Path. Bact. 32, 15.

Frank, B. (1899). Die bisher erzielten Ergebnisse der Nitraginimpfung. Landw. Vers.Sta. 51, 441.

Hughes, D. Q. \& Vincent, J. M. (1942). Serological studies of the root-nodule bacteria. III. Test of neighbouring strains of the same species. Proc. Linn. Soc. N.S.W. 67, 1942.

Hutchinson, C. M. (1924). Report of Imperial Agricultural Bacteriologist. III. Soil . biology. Sci. Rep. agric. Res. Inst. Pusa, 32.

Kueczkowska, J. (1945 a). The production of plaques by Rhizobium bacteriophage in poured plates and its value as a counting method. J. Bact. 50, 71 .

KIECZKOWsKA, J. (1945b). A quantitative study of the interaction of bacteriophage with Rhizobium using the technique of poured plates. $J$. Bact. 50, 81 .

Krasir'NIKov, N. A. (1941).Variability of nodule bacteria. I. The origin of strains under the influence of bacteriophage. Mikrobiologia, 10 (4), 396.

LURIA, S. E. \& DeLBRück, M. (1943). Mutations of bacteria from virus sensitivity to virus resistance. Genetics, $28,491$.

Newcombe, H. B. (1949). Origin of bacterial variants. Nature, Lond., 164, 150.

Nicol, H. \& ThonNTon, H. G. (1941). Competition between related strains of nodule bacteria and its influence on infection of the legume host. Proc. Roy. Soc. B, $130,32$.

NoBBe, F. \& HrLrNer, L. (1893). Wodurch werden die Knöllchenbesitzeden Leguminosen befähigt den freien atmospharishen Stickstoff für sich zu verwerten? Landw. Vers.Sta. 42, 459.

NutMan, P. S. (1946). Variation within strains of clover nodule bacteria in the size of nodule produced and the 'effectivity' of the symbiosis. J. Bact. 57, 41. 
Simon, J. (1908). Die Widerstandsfähigheit des Wurzelbakterien der Leguminosen und ihre Bedeutung für die Bodenimpfung. Angew. Bot. 5, 132.

Snieszko, S. (1929). Beitrage zur Kenntnis der Zelluloze zersetzenden Bakterien. Zbl. Bakt. Abt. II, 78, 375.

StAPP, C. (1929). Zur Frage der Planmässigen Erteilung hochwirksamer LeguminosenKnöllchenbakterienkulturen. Angerv. Bot. 11, 197.

Virtanen, A. I. (1945). Symbiotic nitrogen fixation. Nature, Lond., 155, 747.

(Received 22 October 1949) 